\title{
Experimental Evidence for the Quasi-“Living" Nature of the Grignard Metathesis Method for the Synthesis of Regioregular Poly(3-alkylthiophenes)
}

\author{
Mihaela Corina Iovu, Elena E. Sheina, Roberto R. Gil, and \\ Richard D. McCullough*
}

Department of Chemistry, Carnegie Mellon University, Pittsburgh, Pennsylvania 15213

Received May 31, 2005; Revised Manuscript Received August 15, 2005

\begin{abstract}
The Grignard metathesis (GRIM) polymerization of 3-alkylthiophenes proceeds by a quasi"living" chain growth mechanism, not by a step growth process. Kinetic studies of the Grignard metathesis polymerization of 2,5-dibromo-3-alkylthiophenes showed that the molecular weight of poly(3-alkylthiophenes) is a function of the molar ratio of the monomer to nickel initiator, and conducting polymers with predetermined molecular weights and relatively narrow molecular weight distributions $(\mathrm{PDIs}=$ $1.2-1.5)$ can be made. Sequential monomer addition resulted in new block copolymers containing different poly(3-alkylthiophene) segments.
\end{abstract}

\section{Introduction}

Conventional design of advanced organic materials that display a variety of desirable properties in a controllable way continues to be one of the great challenges of the contemporary polymer research. One class of these advanced materials is organic conducting polymers. Since the initial discovery of these polymers in the late 1970s, various applications of these materials have been explored due to their exceptional electronic and photonic properties. ${ }^{1-3}$

The straightforward synthesis of polythiophene derivatives (PT) generates soluble and processable polymers with a wide range of practical and potential applications, including rechargeable batteries, ${ }^{1}$ electrochromic devices, ${ }^{1}$ chemical and optical sensors, ${ }^{1}$ lightemitting diodes, ${ }^{4-6}$ and field-effect transistors. ${ }^{7}$ While traditional approaches to synthesizing PTs derivatives via electrochemical or oxidative chemical polymerization methods yield polymers with various degrees of regioregularity, ${ }^{3}$ the regioselective synthesis of poly(3-alkylthiophenes) (PATs) results in almost exclusively headto-tail (HT) couplings.

The synthesis of regioregular PATs, first discovered by McCullough et al..$^{8,9}$ and shortly followed by Rieke, ${ }^{10}$ results in the formation of defect-free, structurally homogeneous HT-PATs that have greatly improved electronic and photonic properties compared to regiorandom analogues, ${ }^{11,12}$ including significantly enhanced conductivity. One drawback with the McCullough and Riecke methods lies in their use of cryogenic temperatures. ${ }^{8}$ The discovery of the Grignard metathesis (GRIM) method allows the polymerization to occur at room temperature or at reflux, hence leading to a quick and cost-effective technique for the large-scale synthesis of high molecular weight, regioregular PATs. ${ }^{13,14}$

All of the aforementioned polymerizations are transition-metal-catalyzed cross-coupling reactions. ${ }^{15}$ The generally accepted mechanism for these $\mathrm{Ni}(\mathrm{II})$ catalyzed

\footnotetext{
* Corresponding author. E-mail: rm5g@andrew.cmu.edu.
}

cross-coupling reactions involves a catalytic cycle of three consecutive steps: oxidative addition, transmetalation, and reductive elimination. ${ }^{16-26}$ The course of the catalytic reaction has been extensively studied and proven to be affected by both the ligand structure and the choice of the metal. ${ }^{15}$ It has been recently reported that the nickel-initiated cross-coupling polymerization proceeds via a chain-growth mechanism. ${ }^{27,28}$ Furthermore, the addition of various Grignard reagents $\left(\mathrm{R}^{\prime} \mathrm{MgX}\right)$ at the end of polymerization results in the end-capping of regioregular PATs with an $\mathrm{R}^{\prime}$ end group. ${ }^{29}$

Here we show that the Grignard metathesis polymerization generates regioregular poly(3-alkylthiophenes) with precise molecular weights and very narrow polydispersities. Furthermore, by the sequential addition of different 3-alkylthiophene monomers, this method leads to the synthesis of block copolymers. The near "living" nature of the polymerization also presented and allows for the development of new architectures and the ability to create regioregular poly(3-alkylthiophenes) with specific functionalities.

\section{Experimental Part}

Materials. Synthesis of 2,5-dibromo-3-hexylthiophene and 2,5-dibromo-3-dodecylthiophene were performed according to the literature. ${ }^{13,14}$ Tetrahydrofuran (THF) was dried over $\mathrm{K} /$ benzophenone under nitrogen and freshly distilled prior to use. [1,3-Bis(diphenylphosphino)propane]dichloronickel(II) (98\%) $\left(\mathrm{Ni}(\mathrm{dppp}) \mathrm{Cl}_{2}\right)$, tert-butylmagnesium chloride $(2 \mathrm{M}$ in diethyl ether) and $p$-dimethoxybenzene (98\%) were purchased from Aldrich Chemical Co., Inc., and used without further purification.

Polymerization Kinetic Experiments. In a typical experiment, a dry $100 \mathrm{~mL}$ three-neck round-bottom flask was flushed with $\mathrm{N}_{2}$ and charged with 2,5-dibromo-3-hexylthiophene (1.6 g, $5 \mathrm{mmol}$ ), $p$-dimethoxybenzene (internal standard) $(0.2 \mathrm{~g})$, and anhydrous THF $(50 \mathrm{~mL})$. A $2 \mathrm{M}$ solution of tertbutylmagnesium chloride $(2.5 \mathrm{~mL}, 5 \mathrm{mmol})$ in diethyl ether $\left(\mathrm{Et}_{2} \mathrm{O}\right)$ was added via a deoxygenated syringe, and the reaction mixture was gently refluxed for $2 \mathrm{~h}$. At this time an aliquot $(0.5 \mathrm{~mL})$ was taken out and quenched with water. The organic phase was extracted in $\mathrm{Et}_{2} \mathrm{O}$ analyzed by GC-MS to determine 
the composition of the reaction mixture. The main components of the reaction mixture were 2-bromo-5-chloromagnesium-3hexylthiophene and 5-bromo-2-chloromagnesium-3-hexylthiophene regioisomers. Usually less than $5 \%$ of unreacted 2,5dibromo-3-hexylthiophene was detected by GC-MS analysis. The concentration of 2-bromo-5-chloromagnesium-3-hexylthiophene isomer was considered as the initial monomer concentration. The oil bath was then removed, and the reaction mixture was allowed to cool to $23-25{ }^{\circ} \mathrm{C}$, at which time $\mathrm{Ni}(\mathrm{dppp}) \mathrm{Cl}_{2}$ (0.04 g, $\left.0.075 \mathrm{mmol}\right)$ was added as a suspension in $1 \mathrm{~mL}$ of anhydrous THF. After addition of $\mathrm{Ni}(\mathrm{dppp}) \mathrm{Cl}_{2}$, aliquots $(1 \mathrm{~mL})$ were taken at different time intervals, and each was precipitated in methanol $(5 \mathrm{~mL})$. For each aliquot a sample was prepared in $\mathrm{Et}_{2} \mathrm{O}(2 \mathrm{~mL})$ and analyzed by GC-MS for the determination of concentration of unreacted monomer. After filtration through PTFE filters $(0.45 \mu \mathrm{m})$, the molecular weight of the pristine polymer samples was measured by GPC.

Chain Extension Experiment for the Synthesis of Poly(3-hexylthiophene)-b-poly(3-dodecylthiophene). A dry $250 \mathrm{~mL}$ three-neck round-bottom flask (A) was charged with 2,5-dibromo-3-hexylthiophene (1.6 g, $5 \mathrm{mmol}$ ), $p$-dimethoxybenzene (internal standard) ( $0.3 \mathrm{~g})$, and anhydrous THF (165 $\mathrm{mL})$. A $2 \mathrm{M}$ solution of tert-butylmagnesium chloride $(2.5 \mathrm{~mL}$, $5 \mathrm{mmol})$ in diethyl ether $\left(\mathrm{Et}_{2} \mathrm{O}\right)$ was added via a deoxygenated syringe, and the reaction mixture was gently refluxed for $2 \mathrm{~h}$. After the consumption of 2,5-dibromo-3-hexylthiophene the reaction mixture was cooled at $20-22{ }^{\circ} \mathrm{C}$. The concentration of unreacted 2-bromo-5-chloromagnesium-3-hexylthiophene was determined by GC-MS (more than $90 \%$ of monomer was consumed in $2 \mathrm{~h})$. $\mathrm{Ni}(\mathrm{dppp}) \mathrm{Cl}_{2}(0.05 \mathrm{~g}, 0.1 \mathrm{mmol})$ was added as a suspension in $1 \mathrm{~mL}$ of anhydrous THF. The polymerization continued for $3 \mathrm{~h}$ before addition of 2-bromo-5-chloromagnesium-3-dodecylthiophene (prepared as described below).

A dry $50 \mathrm{~mL}$ three-neck round-bottom flask (B) flushed with $\mathrm{N}_{2}$ was charged with 2,5-dibromo-3-dodecylthiophene (4.1 g, $10 \mathrm{mmol})$ and anhydrous THF (10 mL). A $2 \mathrm{M}$ solution of tertbutylmagnesium chloride $(5 \mathrm{~mL}, 10 \mathrm{mmol})$ in diethyl ether $\left(\mathrm{Et}_{2} \mathrm{O}\right)$ was added via a deoxygenated syringe, and the reaction mixture was gently refluxed for $2 \mathrm{~h}$. The concentration of 2-bromo-5-chloromagnesium-3-dodecylthiophene was determined by GC-MS.

Analyses. GC-MS analysis was performed on a HewlettPackard Agilent 6890-5973 GC-MS workstation. The GC column was a Hewlett-Packard fused silica capillary column cross-linked with 5\% phenylmethylsiloxane. Helium was the carrier gas. The following conditions were used for all GC-MS analyses: injector temperature, $250{ }^{\circ} \mathrm{C}$; initial temperature, $70{ }^{\circ} \mathrm{C}$; temperature ramp, $10{ }^{\circ} \mathrm{C} / \mathrm{min}$; final temperature, 300 ${ }^{\circ} \mathrm{C}$. Gel permeation chromatography (GPC) measurements were performed on a Waters 2690 separations module apparatus and a Waters 2487 dual $\lambda$ absorbance detector with chloroform as the eluent (flow rate $1 \mathrm{~mL} / \mathrm{min}, 35^{\circ} \mathrm{C}, \lambda=254$ $\mathrm{nm})$ and a series of three Styragel columns (104, 500, $100 \AA$; Polymer Standard Services). Toluene was used as an internal standard, and calibration based on polystyrene standards was applied for determination of molecular weights. ${ }^{1} \mathrm{H}$ NMR spectra of the polymer solutions in $\mathrm{CDCl}_{3}$ were recorded on a Bruker Avance $500 \mathrm{MHz}$ spectrometer. UV-vis-NIR spectra were measured on polymer solutions in anhydrous chloroform or polymer thin films cast onto $22 \mathrm{~mm}$ square cover glass using a UV-vis-NIR spectrophotometer (Varian Cary 5000). Electrical conductivity measurements were performed by a standard spring-loaded pressure contact Signatone S-301-4 fourpoint probe, which was connected to a Hewlett-Packard 6632A system dc power supply, a Hewlett-Packard 3457 A multimeter (for voltage measurements), and a Keithley model 196 system DMM (for current measurements). Polymer films for conductivity measurements were prepared by drop-casting from toluene solutions. The casting solutions were prepared by dissolving $5 \mathrm{mg}$ of polymer in $1 \mathrm{~mL}$ of dry toluene and sonicating for $5 \mathrm{~min}$, followed by the filtration of clear solutions through PTFE $0.45 \mu \mathrm{m}$ filters. Films were drop-cast onto 22 $\mathrm{mm}$ square cover glass that were washed with chromic acid solution, rinsed several times with acetone and hexanes, and dried before drop-casting. Conductivities were measured for

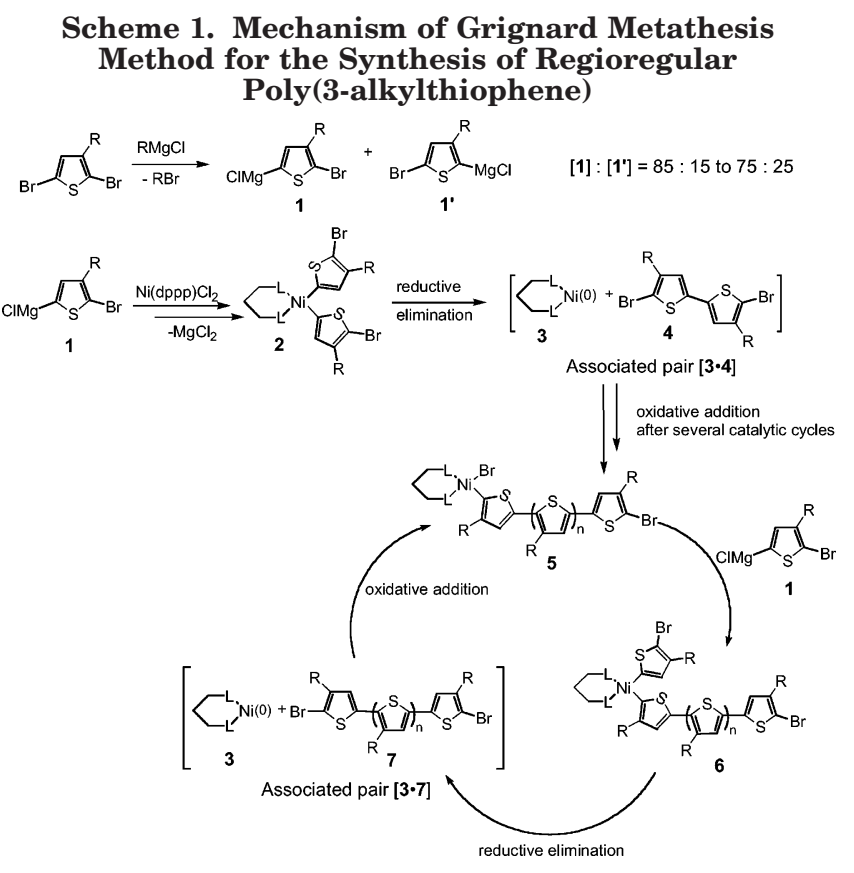

films of the polymers oxidized with $\mathrm{I}_{2}$ for different times $(1 \mathrm{~h}$ is the usual doping time). The film thickness was measured by scanning electron microscopy (SEM) and the conductivity calculated according to the following equation:

$$
\sigma=\frac{1}{4.53 R l}
$$

where $R$ is the resistance $(R=V / I)$ and $l$ is the film thickness. The film thickness (cross section) was measured by SEM using a Hitachi S-2460N electron microscope.

\section{Results and Discussion}

The discovery of nickel-catalyzed aryl-aryl bond formations of Grignard reagents with organohalides by Kumada $^{16,17}$ and Corriu ${ }^{30}$ has led to a significant development in the synthesis of various types of thiophenes. Consequently, the Kumada reaction has been applied to the synthesis of oligothiophenes and polythiophenes. Despite its common use, the mechanism of the nickel-catalyzed cross-coupling polymerization has not been fully understood. Historically, three different mechanistic pathways have been independently proposed. ${ }^{16-26}$ We will focus on the mechanistic pathway suggested by Negishi, ${ }^{18,19}$ Yamamoto, ${ }^{20-22}$ and Parshall, ${ }^{23}$ which was later extended to cross-coupling polycondensation. ${ }^{31,32}$ It has been proposed that the reductive elimination and oxidative addition were stepwise processes, which involved formation of a "free" $\mathrm{Ni}(0)$ intermediate, with the transmetalation as the rate-determining step. The experimental observations from both McCullough and Grignard metathesis methods has led to a new mechanistic proposal for the nickelcatalyzed cross-coupling polymerization for the synthesis of regioregular poly(3-alkylthiophenes). ${ }^{16-26}$

We have recently proposed the mechanism of the nickel-initiated cross-coupling polymerization for the synthesis of regioregular poly(3-alkylthiophene) (McCullough method). ${ }^{27}$ The proposed mechanism of Grignard metathesis is shown in Scheme 1. In the first reaction of Scheme 1, treatment of 2,5-dibromo-3alkylthiophene with 1 equiv of $\mathrm{RMgCl}(\mathrm{R}=$ alkyl) results in magnesium-bromine exchange reaction, also referred to as Grignard metathesis (GRIM). This reac- 


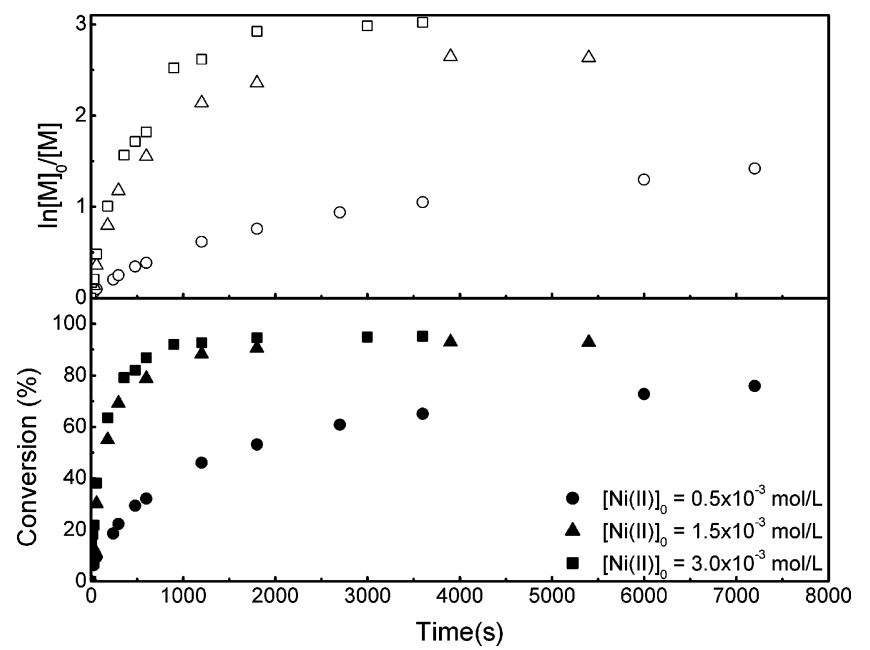

Figure 1. Conversion (filled symbols) and logarithm of monomer concentration (open symbols) vs time plots for 2-bromo-5-chloromagnesium-3-hexylthiophene Grignard metathesis polymerization at variable $\mathrm{Ni}(\mathrm{dppp}) \mathrm{Cl}_{2}$ concentrations; $[\mathrm{M}]_{0}=0.07 \mathrm{~mol} / \mathrm{L} ; \mathrm{THF} ; 23-25{ }^{\circ} \mathrm{C}$.

tion proceeds with a moderate degree of regioselectivity, leading to a distribution of regiochemical isomers $\mathbf{1}$ and $\mathbf{1}^{\prime}$ of $85: 15$ to $75: 25$. Lowering the temperature during magnesium halogen exchange reaction results in the formation of isomer $\mathbf{1}$ in higher proportion ([1]:[1'] = $85: 15)$ when the reaction is performed at room temperature. To verify the incorporation of isomer 1, we needed to analyze the reaction mixture. The GC-MS analysis of the quenched reaction mixture, after the addition of $\mathrm{Ni}(\mathrm{dppp}) \mathrm{Cl}_{2}$, indicated that only isomer $\mathbf{1}$ is incorporated into the polymer, while isomer $\mathbf{1}^{\prime}$ was not consumed.

The first step in the mechanism is the reaction of 2 equiv of 2-bromo-5-chloromagnesium-3-alkylthiophene monomer (1) with $\mathrm{Ni}(\mathrm{dppp}) \mathrm{Cl}_{2}$ generating a bis(organo)nickel compound (2), which undergoes reductive elimination resulting in the formation of an associated pair of the 5,5'-dibromobithienyl (tail-to-tail coupling) and $\mathrm{Ni}(0)[3-4]$. We propose that the associated pair is formed by coordination of 1,3-bis(diphenylphosphino)propane-nickel $(0)$ to the thiophene ring in a $\eta^{2}$ - or $\eta^{4}$ bonded fashion. A similar type of $\mathrm{Ni}(0)-\eta^{2}$ arene complex was previously reported..$^{33-35}$ The dimer undergoes a fast oxidative addition to the nickel(0) center, generating a new organonickel compound. Growth of the polymer chain occurs by insertion of one monomer at a time, in which the $\mathrm{Ni}(\mathrm{dppp})$ moiety is incorporated into the polymer chain as an end group (polymer 5 in Scheme 1). According to the proposed mechanism, only one structural defect (one tail-to-tail coupling) per polymer chain is generated during the proposed catalytic cycle.

In the Grignard metathesis method, poly(3-alkylthiophenes) with relatively high molecular weight are produced early in the reaction. ${ }^{27}$ This contradicts the accepted step growth polymerization mechanism proposed for nickel-catalyzed cross-coupling polymerization, in which one expects the fast disappearance of the monomer and increase of the molecular weight toward the end of the reaction. Therefore, we explored the kinetics of the polymerization reaction to achieve a near "living" system.

Influence of $\mathbf{N i}\left(\right.$ dppp) $\mathrm{Cl}_{2}$ Concentration. Several experiments were performed at various $\mathrm{Ni}(\mathrm{dppp}) \mathrm{Cl}_{2}$

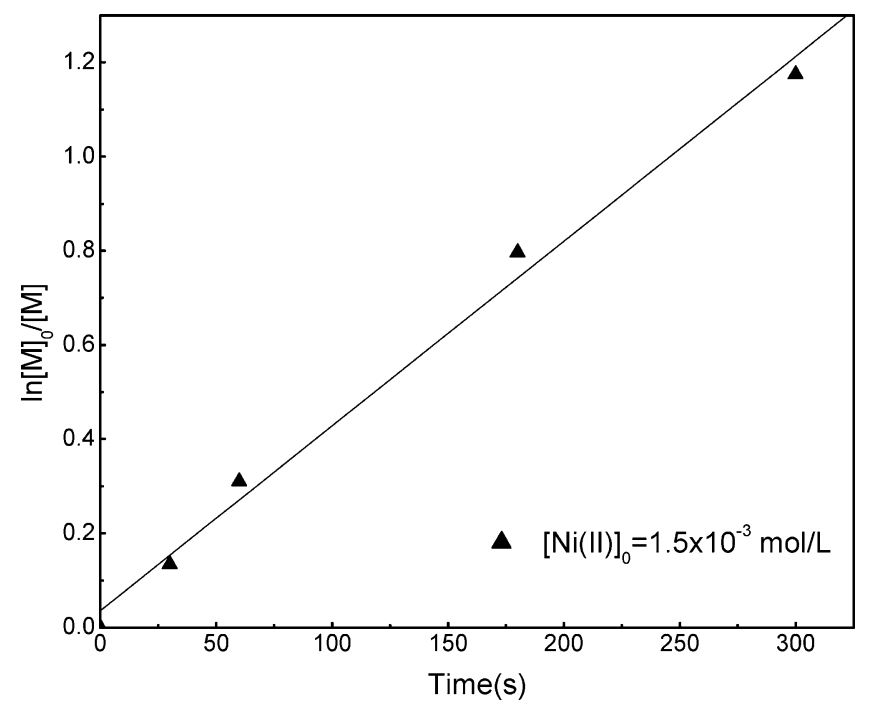

Figure 2. Logarithm of monomer concentration vs time plot for 2-bromo-5-chloromagnesium-3-hexylthiophene Grignard metathesis polymerization; $[\mathrm{M}]_{0}=0.07 \mathrm{~mol} / \mathrm{L}$; THF; $23-25$ ${ }^{\circ} \mathrm{C}$.

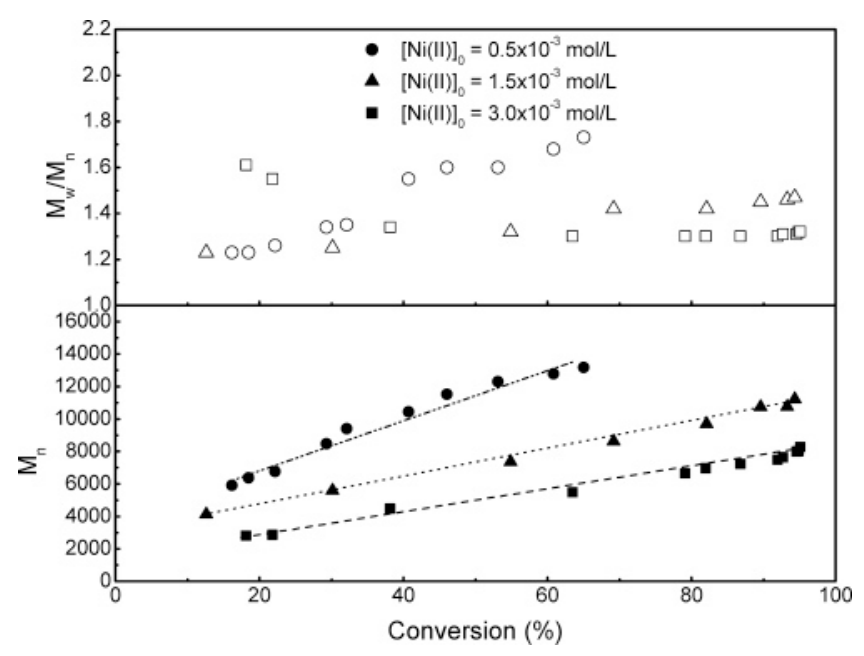

Figure 3. Dependence of molecular weights and polydispersities on conversion for 2-bromo-5-chloromagnesium-3-hexylthiophene Grignard metathesis polymerization at variable $\mathrm{Ni}(\mathrm{dppp}) \mathrm{Cl}_{2}$ concentrations; $[\mathrm{M}]_{0}=0.07 \mathrm{~mol} / \mathrm{L} ; \mathrm{THF} ; 23-25$ ${ }^{\circ} \mathrm{C}$.

concentrations with a constant monomer 1 concentration. As shown in Figure 1, the reaction rates increased with the increase in the $\mathrm{Ni}(\mathrm{dppp}) \mathrm{Cl}_{2}$ concentration. The plots indicate the polymerization is over within just few minutes. The semilogarithmic kinetic plots are linear up to $\sim 60 \%$ conversion (Figure 2 ). The nonlinearity in the semilogarithmic kinetic plots in the late stage of the reaction indicates the presence of termination, which may be due to the aggregation of polymer chains. ${ }^{36}$ If the reaction medium becomes heterogeneous due to the formation of polymer aggregates, the active centers are not accessible for further insertion of the monomer. Because of the nonlinearity of the semilogarithmic kinetic plots, further optimization in the reaction conditions is needed to result in a true "living" GRIM polymerization.

Molecular weight vs conversion plot (Figure 3) and the GPC traces (Figure 4) show the increase of molecular weight with conversion, which supports a "living" chain growth mechanism for nickel-initiated crosscoupling polymerization. The molecular weight of the 


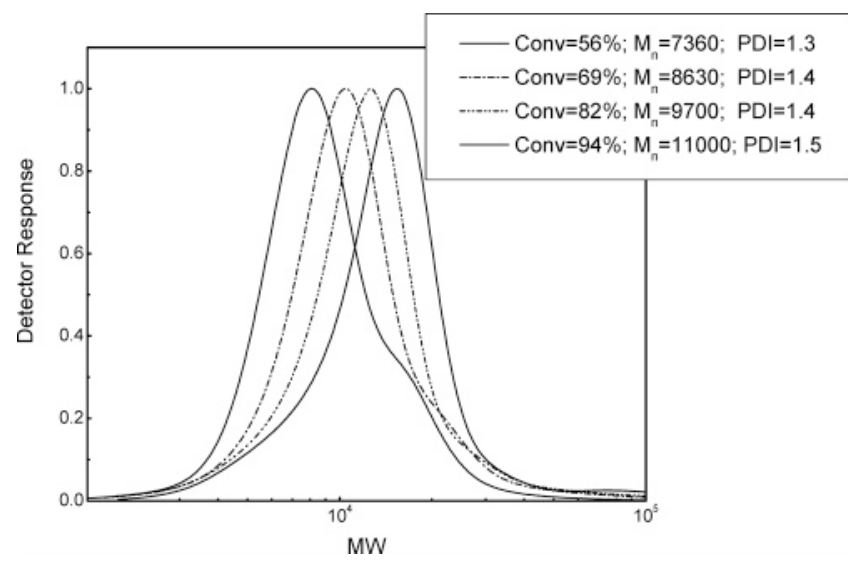

Figure 4. Gel permeation chromatography traces (UV detector) for 2-bromo-5-chloromagnesium-3-hexylthiophene Grignard metathesis polymerization $[\mathrm{Ni}(\mathrm{II})]_{0}=1.5 \times 10^{-3} \mathrm{~mol} / \mathrm{L}$; $[\mathrm{M}]_{0}=0.07 \mathrm{~mol} / \mathrm{L} ; \mathrm{THF} ; 23-25^{\circ} \mathrm{C}$.

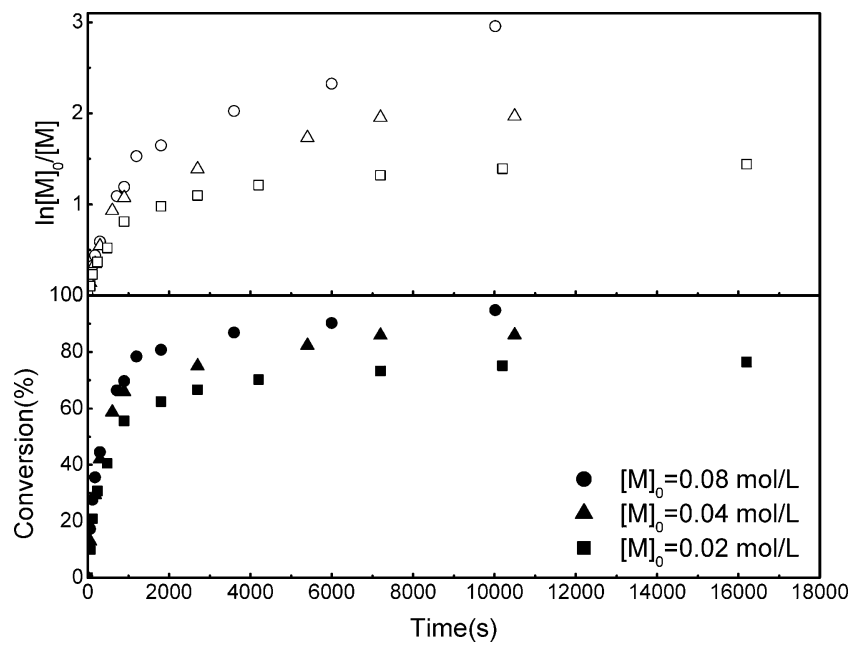

Figure 5. Conversion (filled symbols) and logarithm of monomer concentration (open symbols) vs time plots for Grignard metathesis polymerization at different 2-bromo-5chloromagnesium-3-hexylthiophene concentrations; $[\mathrm{Ni}(\mathrm{II})]_{0}=$ $1.5 \times 10^{-3} \mathrm{~mol} / \mathrm{L}$; THF; $0-2{ }^{\circ} \mathrm{C}$.

polymers is a function of the molar ratio of monomer 1 to $\mathrm{Ni}(\mathrm{dppp}) \mathrm{Cl}_{2}$ initiator (Figure 3). Furthermore, the number-average molecular weight of the polymers can be predicted by the following formula:

$$
\mathrm{DP}_{\mathrm{n}}=\frac{\Delta[\mathrm{M}]_{t}}{\left[\mathrm{Ni}(\mathrm{dppp}) \mathrm{Cl}_{2}\right]_{0}}
$$

According to our proposed mechanism, $\mathrm{Ni}(\mathrm{dppp}) \mathrm{Cl}_{2}$ acts as an initiator rather than a catalyst, and the $\mathrm{Ni}(\mathrm{dppp})$ moiety is incorporated in the polymer as an end group (polymer 2, Scheme 1). Poly(3-hexylthiophenes) with relatively narrow polydispersities (as low as PDI $=1.2$ ) were obtained for the experiments performed at higher concentration of $\mathrm{Ni}(\mathrm{dppp}) \mathrm{Cl}_{2}$ (Figure 3 ).

Influence of Monomer Concentration. To complement the previous experiments, another series of experiments were conducted at a constant $\mathrm{Ni}(\mathrm{dppp}) \mathrm{Cl}_{2}$ concentration, while varying the 2-bromo-5-chloromagnesium-3-hexylthiophene (monomer) concentration. These experiments were performed in order to determine the reaction order with respect to the monomer. The polymerizations were conducted at low temperatures $\left(0-2{ }^{\circ} \mathrm{C}\right)$ to slow the rate of reaction and conserve

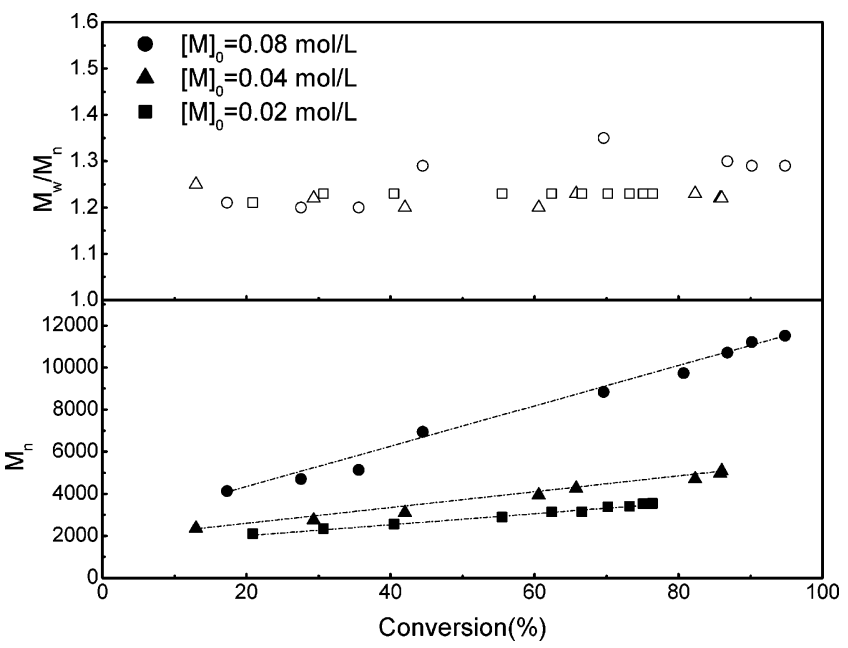

Figure 6. Dependence of molecular weights and polydispersities on conversion for Grignard metathesis polymerization at different 2-bromo-5-chloromagnesium-3-hexylthiophene concentrations; $[\mathrm{Ni}(\mathrm{II})]_{0}=1.5 \times 10^{-3} \mathrm{~mol} / \mathrm{L} ; \mathrm{THF} ; 0-2{ }^{\circ} \mathrm{C}$.

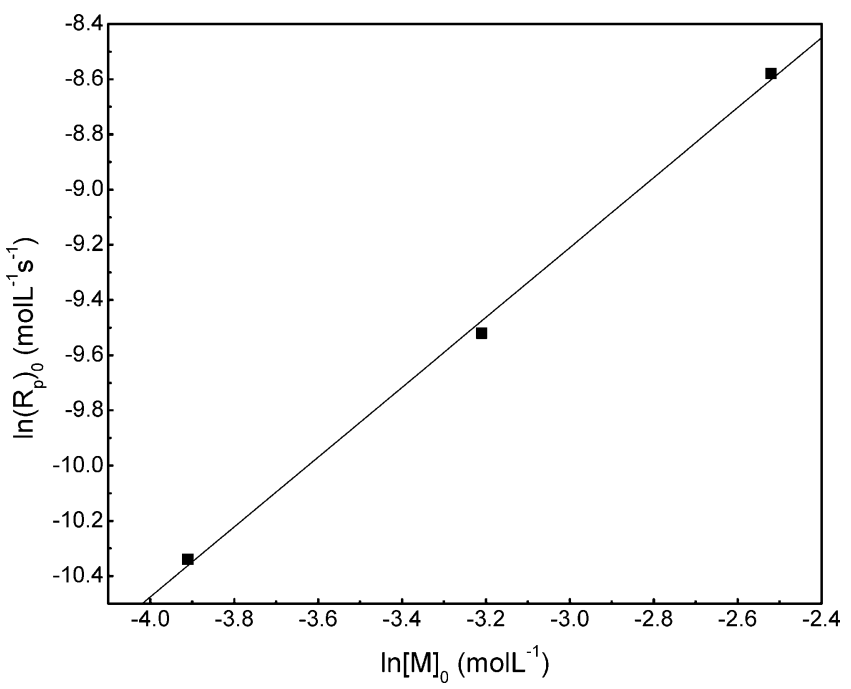

Figure 7. Plot of the logarithm of the initial rate of polymerization vs logarithm of monomer concentration for Grignard metathesis polymerization of 2-bromo-5-chloromagnesium-3hexylthiophene; $[\mathrm{Ni}(\mathrm{II})]_{0}=1.5 \times 10^{-3} \mathrm{~mol} / \mathrm{L} ; \mathrm{THF} ; 0-2{ }^{\circ} \mathrm{C}$.

the linearity of the semilogarithmic kinetic plots. The latter allowed for a more accurate determination of the initial polymerization rate. As shown in Figure 5, the reaction rates increased with the increase in the monomer concentration.

Figure 6 shows the increase of molecular weight with conversion. Poly(3-hexylthiophenes) with relatively narrow polydispersities (PDI < 1.5) were synthesized.

A value of $\sim 1$ for the reaction order with respect to the monomer was obtained from the slope of the plot of the logarithm of the initial rate of polymerization vs the logarithm of the monomer concentration (Figure 7).

End-Group Analysis of Regioregular Poly(3hexylthiophene). Grignard metathesis method for the synthesis of poly(3-alkylthiophenes) results in the formation of regioregular polymers $(\sim 98 \%$ head-to-tail couplings). Quenching of nickel terminated poly(3alkylthiophene) (polymer 2, Scheme 1) with methanol/ $\mathrm{HCl}$ mixture results in the formation of $\mathrm{H} / \mathrm{Br}$ terminated polymer. According to the mechanism proposed here, only one tail-to-tail coupling should occur in the regioregular polymerization of 3-alkylthiophenes. Here we 

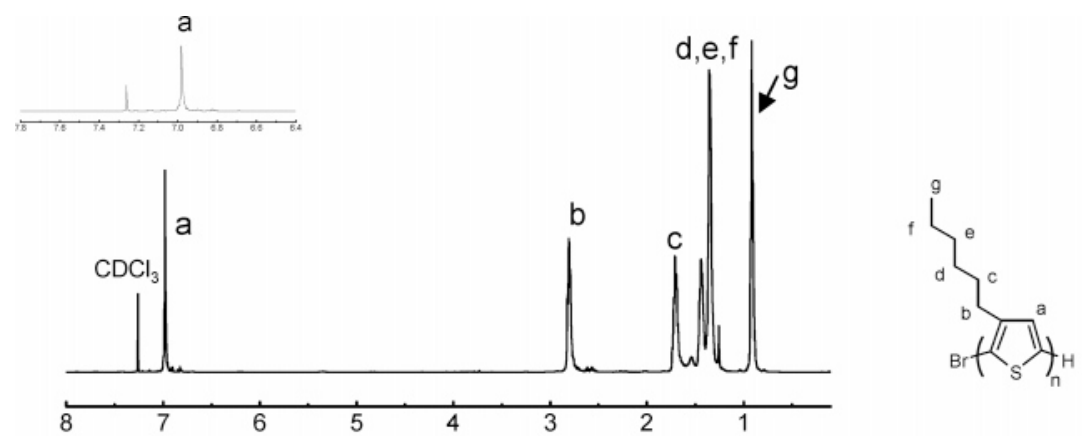

a
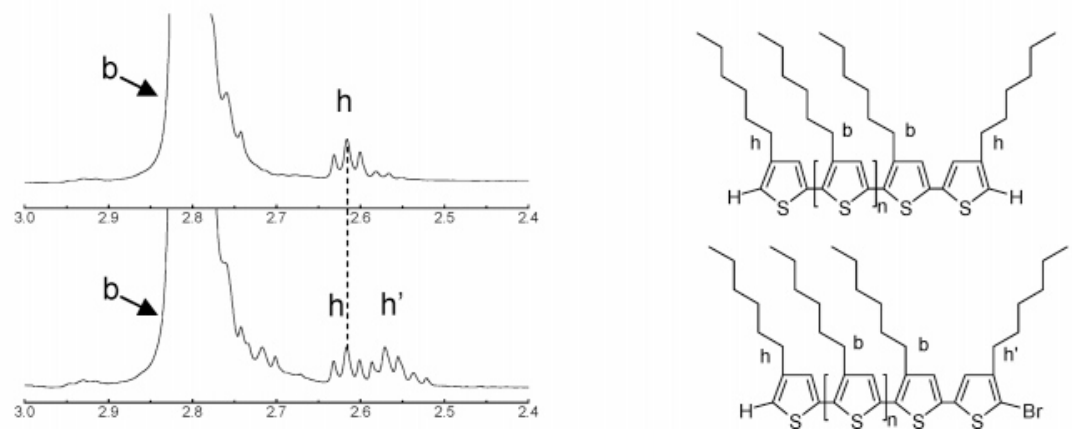

b

C

Figure 8. ${ }^{1} \mathrm{H}$ NMR (500 MHz) spectra of (a) rr-poly(3-hexylthiophene) H/Br terminated; (b) expansion of rr-poly(3-hexylthiophene) $\mathrm{H} / \mathrm{H}$ terminated; and (c) expansion of rr-poly(3-hexylthiophene) $\mathrm{H} / \mathrm{Br}$ terminated.

Scheme 2. Magnesium Halogen Exchange of $\mathrm{H} / \mathrm{Br}$ Terminated Regioregular Poly(3-hexylthiophene)

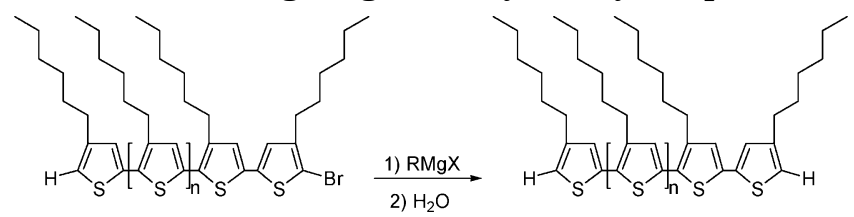

Table 1. Integration Values from the ${ }^{1} \mathrm{H}$ NMR (500 MHz) Spectrum of rr-Poly(3-hexylthiophene) (PHT) H/Br and H/H Terminated

\begin{tabular}{ccc}
\hline peak & PHT-H/Br & PHT-H/H \\
\hline $\mathbf{b}$ & 1 & 1 \\
$\mathbf{h}$ & 0.02 & 0.04 \\
$\mathbf{h}^{\prime}$ & 0.02 &
\end{tabular}

investigate the structures of the resulting polymers by NMR spectroscopy.

As an example, the full $500 \mathrm{MHz}{ }^{1} \mathrm{H}$ NMR spectrum of a moderate molecular weight regioregular poly(3hexylthiophene) (rr-PHT) is presented in Figure 8a. The main absorption signals of rr-PHT are assigned as shown. Two small triplets at $\delta \sim 2.6 \mathrm{ppm}$ of the same intensity for $\mathrm{H} / \mathrm{Br}$ terminated rr-PHT can be assigned to the methylene protons on the first carbon substituent (h and $\mathbf{h}^{\prime}$ ) on the end units. Furthermore, the appearance of the two separate triplet signals at different resonance frequencies is due to different chemical environment around $\mathbf{h}$ and $\mathbf{h}^{\prime}$ (Figure 8c). When the $\mathrm{H} / \mathrm{Br}$ terminated polymer is subjected to a magnesium halogen exchange reaction (Scheme 2) and quenched with an acidic methanol/water mixture, a pristine $\mathrm{H} / \mathrm{H}$ terminated rr-PHT is formed. Consequently, the signal generated by the methylene protons $\mathbf{h}^{\prime}$ is shifted downfield with the two groups (h and $\mathbf{h}^{\prime}$ ) resonating at the same frequency (Figure $8 b$ ). Table 1 presents the integration values from the ${ }^{1} \mathrm{H}$ NMR $(500 \mathrm{MHz})$ spectrum of rr-PHT-H/Br and rr-PHT-H/H terminated polymers. Note that the intensity of the $\mathbf{h}$ peak is doubled in the absence of the bromine atom relative to the main peak (b) of first $\beta$-substituent methylene protons. These observations indicate that NMR analysis can distinguish between the two different types of coupling (e.g., head-to-tail (HT) and tail-to-tail (TT)), when rr-PHT $\mathrm{H} / \mathrm{H}$ terminated contains only one structural defect per polymer chain. This finding is in full support of the mechanism in Scheme 1 showing that the first step is a tail-to-tail coupling, followed by Ni-initiated polymerization. The NMR cannot distinguish the $\mathrm{H} / \mathrm{H}$ terminated methylene resonances. However, it allows a relatively accurate determination of molecular weight from the integration of end-group resonances relative to the bulk polymer. For instance, $\mathrm{DP}_{\mathrm{n}}$ for the aforementioned polymer is equal to the ratio of $\mathbf{b}$ to $\mathbf{h}$ and results in 50 monomer units corresponding to $M_{\mathrm{n}}=$ 8300.

Chain Extension by Sequential Monomer Addition. Synthesis of Poly(3-hexylthiophene)-b-poly(3-dodecylthiophene). A previous report from our group showed that the addition of various Grignards reagents to the nickel terminated poly(3-alkylthiophene) results in the formation of end-functional polymers. ${ }^{29}$ Addition of a new portion of 2-bromo-5-chloromagnesium-3-alkylthiophene monomer at the end of the polymerization also resulted in the further increase of the molecular weight of the final polymer. Both experiments strongly indicate the "living" nature of the nickel terminated poly(3-alkylthiophene).

The main focus of this section is the chain extension of rr-PATs through the sequential addition of a different monomer. An example of chain extension is based on the synthesis of poly(3-hexylthiophene)- $b$-poly(3-dodecylthiophene) (PHT-b-PDDT) block copolymer and is described herein for the first time.

The synthetic strategy used for the synthesis of PHT$b$-PDDT is outlined in Scheme 3.

The first step involves preparation of rr-PHT with well-defined end group and structural homogeneity. The reaction parameters were chosen with a special consid- 
Scheme 3. Synthesis of Poly(3-hexylthiophene)-b-poly(3-dodecylthiophene) by Chain Extension through Sequential Monomer Addition

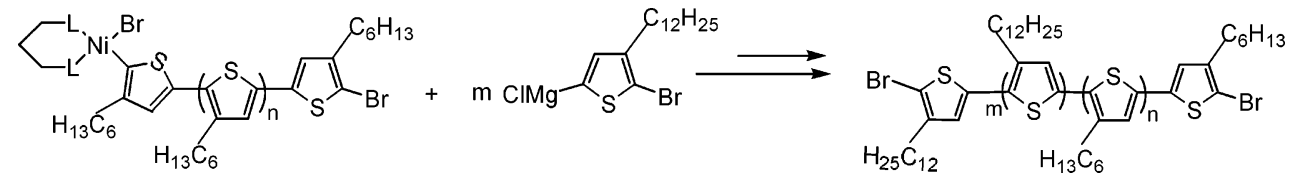

eration with respect to the monomer (2,5-dibromo-3hexylthiophene) concentration (e.g., $[\mathrm{HT}]_{0}=0.02 \mathrm{~mol} /$ L). Higher dilutions are preferable to ensure easier access to the active centers and to avoid possible precipitation of the resultant polymer. The second step of the reaction is preparation of 2-bromo-5-chloromagnesium-3-dodecylthiophene using Grignard metathesis. This reaction generates a mixture of regiochemical isomers with a typical distribution in the range of 75:25 to $85: 15$. The 5-bromo-2-chloromagnesium-3dodecylthiophene does not participate in the catalytic cycle due to the sterically hindered 2-position. The

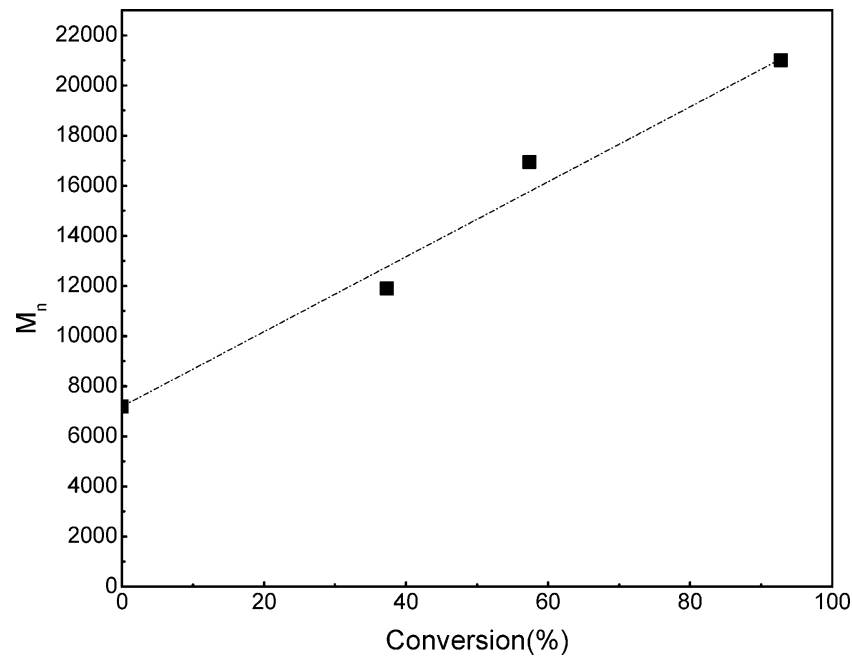

Figure 9. Molecular weight vs conversion plot for Grignard metathesis polymerization of 2-bromo-5-chloromagnesium-3dodecylthiophene initiated by nickel terminated poly (3-hexylthiophene); $\left[\mathrm{M}_{\mathrm{HT}}\right]_{0}=0.02 \mathrm{~mol} / \mathrm{L} ;[\mathrm{Ni}(\mathrm{II})]_{0}=0.6 \times 10^{-3} \mathrm{~mol} / \mathrm{L}$; $\left[\mathrm{M}_{\mathrm{DT}}\right]_{0}=0.04 \mathrm{~mol} / \mathrm{L} ; \mathrm{THF} ; 18-20{ }^{\circ} \mathrm{C}$.

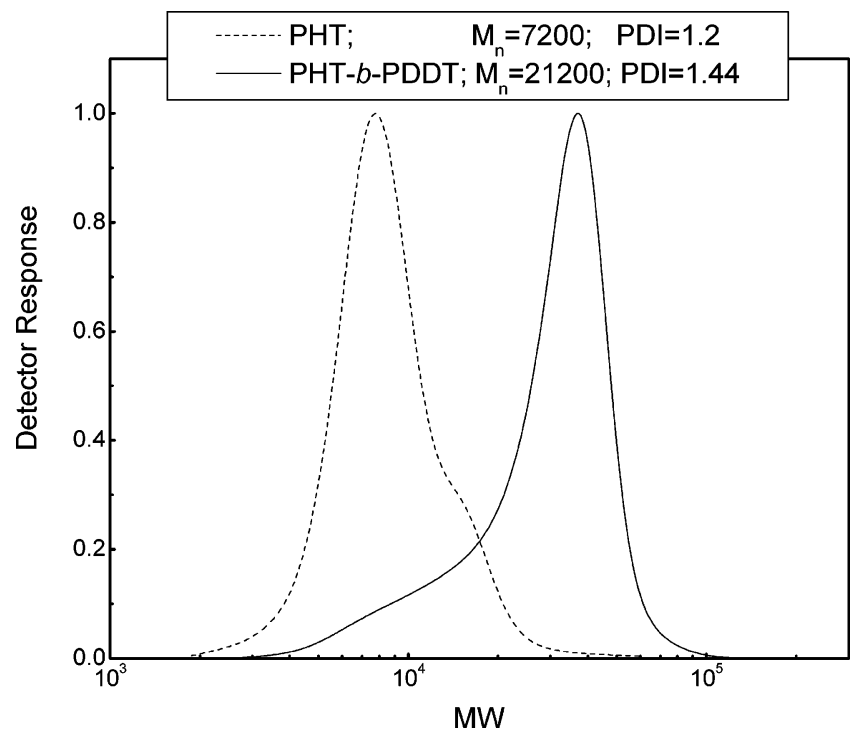

Figure 10. Gel permeation chromatography traces (UV detector) of poly(3-hexylthiophene) and poly(3-hexylthiophene)$b$-poly(3-dodecylthiophene) (chain extension) (reaction conditions listed in Figure 7). reaction was relatively fast and very efficient, reaching $90 \%$ conversion in less than $3 \mathrm{~h}$, at room temperature. The molecular weight vs conversion plot (Figure 9) and the GPC traces (Figure 10) showed an increase of molecular weight with conversion, which strongly supported the chain extension through "living" intermediates. The low molecular weight tailing observed in the GPC trace of PHT- $b$-PDDT indicates the formation of some dead or inactive chains during the chain extension process. The successive monomer insertion into the second block was monitored by ${ }^{1} \mathrm{H}$ NMR. The full ${ }^{1} \mathrm{H}$ NMR spectra of the initial rr-PHT homopolymer and the resultant diblock copolymer are represented in Figure 11. The main resonance frequencies were assigned as shown. Figure 12 illustrates the progressive incorporation of PDDT block into copolymer (the intensity of $\mathrm{g}^{\prime}$ protons increases as the molecular weight of the PDDT block increases). The resultant copolymer was readily soluble in common organic solvents (e.g., chloro-

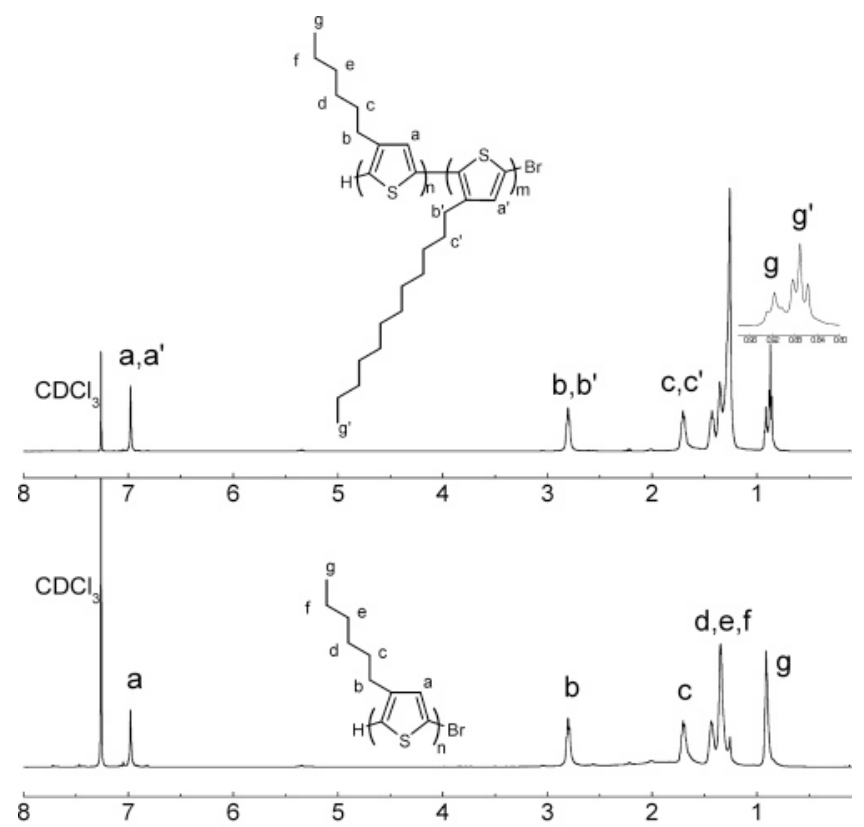

Figure 11. ${ }^{1} \mathrm{H}$ NMR $(500 \mathrm{MHz})$ spectra of poly(3-hexylthiophene) (bottom) and poly(3-hexylthiophene)- $b$-poly(3-dodecylthiophene) (top).

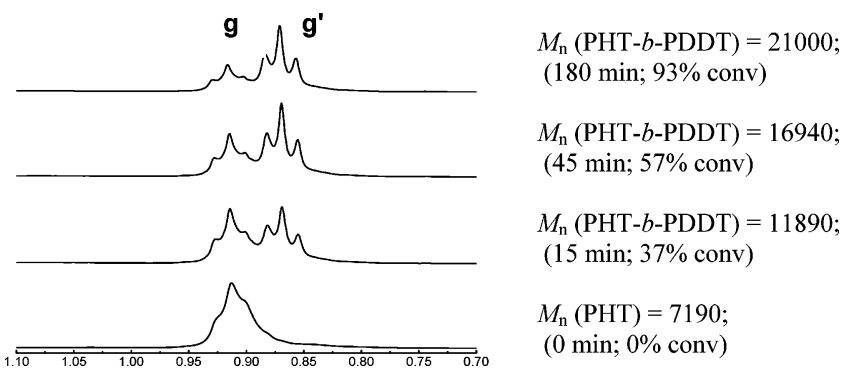

Figure 12. ${ }^{1} \mathrm{H}$ NMR $(500 \mathrm{MHz})$ expansion in the region $0.7-$ $1.1 \mathrm{ppm}$ for starting polymer poly(3-hexylthiophene) and for poly(3-hexylthiophene)- $b$-poly(3-dodecylthiophene) copolymers at different reaction times. 


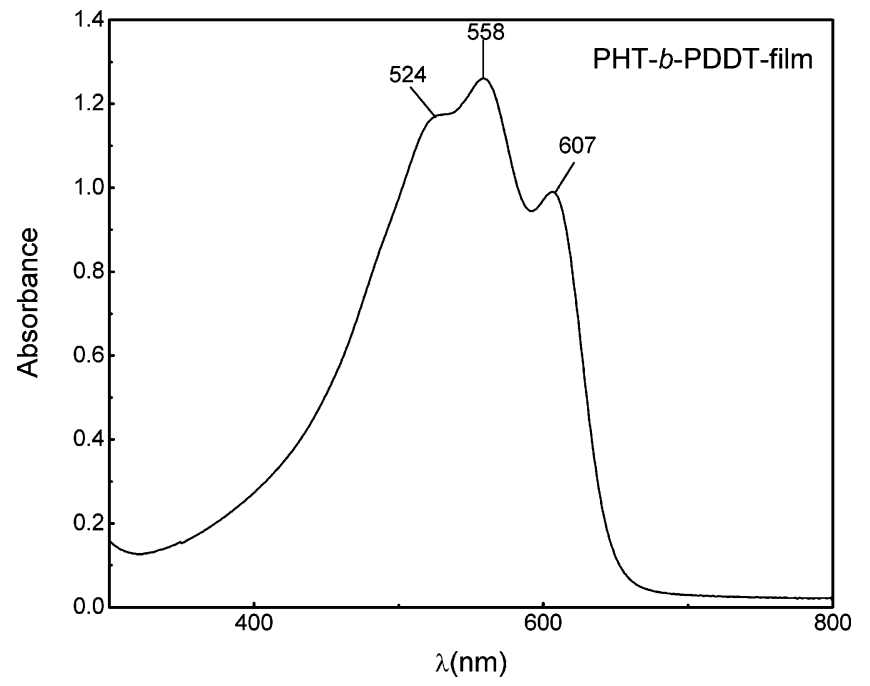

Figure 13. UV-vis spectrum of poly(3-hexylthiophene)- $b$ poly(3-dodecylthiophene) diblock copolymer.

Table 2. Molecular Weights and Polydispersities of Poly(3-dodecylthiophene),

Poly(3-dodecylthiophene)- $b$-polythiophene, and Poly(3-dodecylthiophene)- $b$-polythiophene- $b$ poly(3-dodecylthiophene) (Chain Extension)

\begin{tabular}{lcc}
\hline \multicolumn{1}{c}{ polymer } & $M_{\mathrm{n}}$ & PDI \\
\hline poly(3-dodecylthiophene) & 5120 & 1.19 \\
poly(3-dodecylthiophene)- $b$-polythiophene & 6340 & 1.33 \\
poly(3-dodecylthiophene)- $b$-polythiophene- $b$ - & 9750 & 1.53
\end{tabular}

poly(3-dodecylthiophene $)^{a}$

${ }^{a}$ Reaction conditions: $\left[\mathrm{M}_{\mathrm{DT}}\right]_{0}=0.06 \mathrm{~mol} / \mathrm{L} ;[\mathrm{Ni}(\mathrm{II})]_{0}=1.8 \times$ $10^{-3} \mathrm{~mol} / \mathrm{L} ;\left[\mathrm{M}_{\mathrm{TH}}\right]_{0}=0.02 \mathrm{~mol} / \mathrm{L} ;\left[\mathrm{M}_{\mathrm{DT}}\right]_{1}=0.06 \mathrm{~mol} / \mathrm{L} ; \mathrm{THF} ; 18-$ $20{ }^{\circ} \mathrm{C}$.

form, toluene, 1,2,4-trichlorobenzene, THF) and possessed excellent film-forming abilities.

The UV-vis spectra of the poly(3-hexylthiophene)- $b$ poly(3-dodecylthiophene) (Figure 13) in the solid state indicates the vibrational structure with well-defined peaks (e.g., $\lambda_{\max }=558 \mathrm{~nm}$ and the lower intensity peaks at $\lambda=524$ and $607 \mathrm{~nm}$ ).

Synthesis of Poly(3-dodecylthiophene)-b-polythiophene- $b$-poly(3-dodecylthiophene). Synthesis of poly(3-dodecylthiophene)- $b$-polythiophene- $b$-poly(3dodecylthiophene) triblock copolymer was performed in a similar manner. The length of polythiophene block was carefully chosen due to its very poor solubility. The increase in the molecular weight indicates the formation of the block copolymer (Table 2). However, low molecular weight tailing was observed in the GPC traces, indicating the presence of some dead or inactive chains during the chain extension process. The low solubility of the poly(3-dodecylthiophene)- $b$-polythiophene could cause the precipitation of the nickel terminated polymer, resulting in reduced activity toward further monomer insertion. This could be prevented by the use of higher dilutions or lowering the polythiophene content.

The UV-vis spectra of poly(3-dodecylthiophene) precursor and poly(3-dodecylthiophene)- $b$-polythiophene$b$-poly(3-dodecylthiophene) (Figure 14) films show a bathochromic shift as compared with the solution.

Conductivities of Poly(3-alkylthiophene) Block Copolymers. The films of poly(3-hexylthiophene)- $b$ poly(3-dodecylthiophene) (PHT- $b$-PDDT) and poly(3dodecylthiophene)- $b$-polythiophene- $b$-poly(3-dodecylthiophene) (PDDT- $b$-PT- $b$-PDDT) exhibited relatively

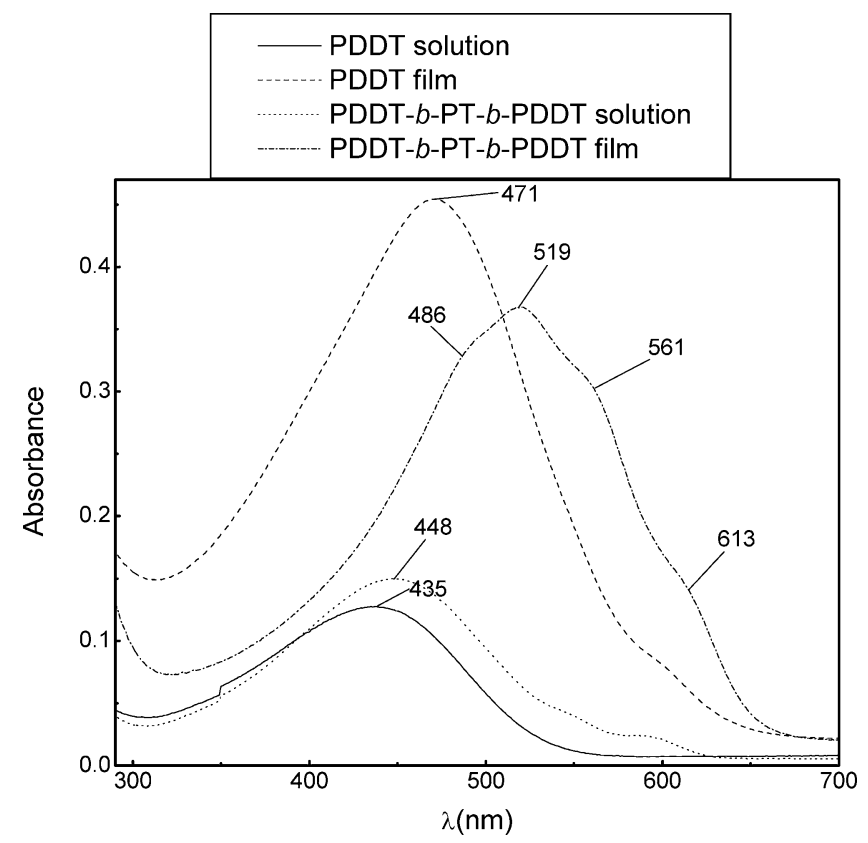

Figure 14. UV-vis spectrum of poly(3-dodecylthiophene) (PDDT) precursor and poly(3-dodecylthiophene)- $b$-polythiophene- $b$-poly(3-dodecylthiophene) (PDDT- $b$-PT- $b$-PDDT) triblock copolymer.

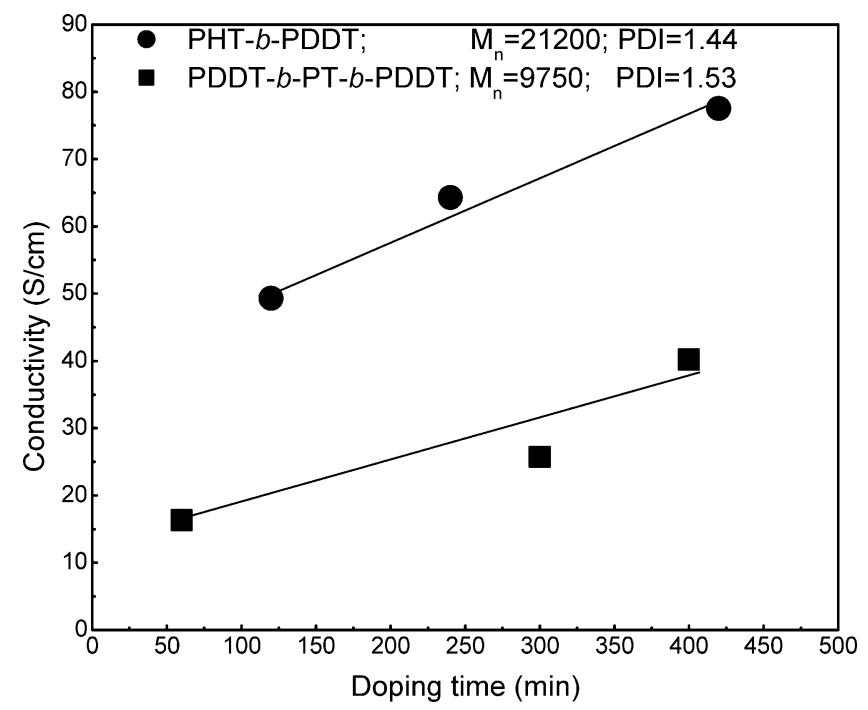

Figure 15. Conductivity of poly(3-hexylthiophene)-b-poly(3dodecylthiophene) and poly(3-dodecylthiophene)- $b$-polythiophene- $b$-poly(3-dodecylthiophene) copolymers as a function of doping time.

good electrical conductivities. As illustrated in Figure 15 , the conductivity increased proportionally with doping times.

\section{Conclusions}

Evidence of the quasi-“living"nature of Grignard metathesis method for the synthesis of regioregular poly(3-alkylthiopehenes) is presented. The kinetic study confirmed that this reaction proceeds via a chain growth mechanism, and the nickel moiety is incorporated in the polymer as an end group. The Grignard metathesis polymerization affords HT PATs with well-defined molecular weight and narrow polydispersities and allows the synthesis of block copolymers by the sequential addition of different 3-alkylthiophene monomers.

The Grignard metathesis method can be now expanded to the synthesis of multiblock copolymers that 
can exhibit enhanced electronic properties, leading to a fabrication of a multitude of novel nano- and microscale electronic materials and devices.

Acknowledgment. We gratefully acknowledge the NSF CHE-0107178 and NSF CHE-0415369 for support of our work. We also thank the following people for helpful discussions: Dr. Malika Jeffries-El, Dr. Darin Laird, Dr. Geneviève Sauvé, and Professor Krzysztof Matyjaszewski.

\section{References and Notes}

(1) Skotheim, T. Handbook of Conducting Polymers; Marcel Dekker: New York, 1986.

(2) Skotheim, T.; Reynolds, J.; Elsembauer, R. Handbook of Conducting Polymers; Marcel Dekker: New York, 1998.

(3) Nalwa, H. S. Handbook of Organic Conductive Molecules and Polymers; J. Wiley \& Sons: New York, 1996.

(4) Heuer, H. W.; Wehrmann, R.; Kirchmeyer, S. Adv. Funct. Mater. 2002, 12, 89-94.

(5) De Paoli, M. A.; Nogueira, A. F.; Machado, D. A.; Longo, C. Electrochim. Acta 2001, 46, 4243-4249.

(6) Groenendaal, L. B.; Zotti, G.; Aubert, P. H.; Waybright, S. M.; Reynolds, J. R. Adv. Mater. 2003, 15, 855-879.

(7) Garnier, F.; Yassar, A.; Hjlaoui, R.; Horowitz, G.; Deloffre, F.; Servet, B.; Ries, S.; Alnot, P. J. Am. Chem. Soc. 1993, 115, 8716-8721.

(8) McCullough, R. D.; Lowe, R. D. J. Chem. Soc., Chem. Commun. 1992, 70-72.

(9) McCullough, R. D.; Lowe, R. D.; Jayaraman, M.; Anderson, D. L. J. Org. Chem. 1993, 58, 904-912.

(10) Chen, T. A.; Wu, X.; Rieke, R. D. J. Am. Chem. Soc. 1995, $117,233-244$.

(11) McCullough, R. D.; Lowe, R. S.; Jayaraman, M.; Ewbank, P C.; Anderson, D. L.; Tristram-Nagle, S. Synth. Met. 1993, 55, 1198-1203.

(12) McCullough, R. D.; Williams, S. P.; Tristram-Nagle, S.; Jayaraman, M.; Ewbank, P. C.; Miller, L. Synth. Met. 1995, $67,279-282$.

(13) Lowe, R. S.; Khersonsky, S. M.; McCullough, R. D. Adv. Mater. 1999, 11, 250-253.

(14) Lowe, R. S.; Ewbank, P. C.; Liu, J.; Zhai, L.; McCullough, R. D. Macromolecules 2001, 34, 4324-4333.
(15) Diederich, F.; Stang, P. J. Metal-Catalyzed Cross-Coupling Reactions; Wiley-VCH: Wernheim, Germany, 1998.

(16) Tamao, K.; Sumitani, K.; Kumada, M. J. Am. Chem. Soc. 1972, 94, 4374-4376.

(17) Kumada, M. Pure Appl. Chem. 1980, 52, 669-679.

(18) Negishi, E. Acc. Chem. Res. 1982, 15, 340-348.

(19) Negishi, E.; Takahashi, T.; Baba, S.; Van Horn, D. E.; Okukado, N. J. Am. Chem. Soc. 1987, 109, 2393-2401.

(20) Yamamoto, A.; Yamamoto, T.; Ozawa, F. Pure Appl. Chem. 1985, 57, 1799-1808.

(21) Ozawa, F.; Hidaka, T.; Yamamoto, T.; Yamamoto, A. J. Organomet. Chem. 1987, 330, 253-263.

(22) Yamamoto, T.; Wakabayashi, S.; Osakada, K. J. Organomet. Chem. 1992, 428, 223-237.

(23) Parshall, G. W. J. Am. Chem. Soc. 1974, 96, 2360-2366.

(24) Morrell, D. G.; Kochi, J. K. J. Am. Chem. Soc. 1975, 97, 72627270 .

(25) Tsou, T. T.; Kochi, J. K. J. Am. Chem. Soc. 1979, 101, 63196332 .

(26) Smith, G.; Kochi, J. K. J. Organomet. Chem. 1980, 198, 199214.

(27) Sheina, E. E.; Liu, J.; Iovu, M. C.; Laird, D. W.; McCullough, R. D. Macromolecules 2004, 37, 3526-3528.

(28) Yokoyama, A.; Miyakoshi, R.; Yokozawa, T. Macromolecules 2004, 37, 1169-1171.

(29) Jeffries-EL, M.; Sauvé, G.; McCullough, R. D. Adv. Mater. 2004, 16, 1017-1019.

(30) Corriu, R. J. P.; Masse, J. P. J. Chem. Soc., Chem. Commun. $1972,144$.

(31) Chen, T. A.; Rieke, R. D. J. Am. Chem. Soc. 1992, 114, 10087-10088.

(32) Chen, T. A.; O’Brien, R. A.; Rieke, R. D. Macromolecules 1993, $26,3462-3463$

(33) Benn, R.; Mynnot, R.; Topalovic, I.; Scott, F. Oraganometallics 1989, 8, 2290-2291.

(34) Scott, F.; Kruger, C.; Betz, P. J. Organomet. Chem. 1990, 387, 113-121.

(35) Browning, J.; Green, M.; Penfold, B. R.; Spencer, J. L.; Stone, F. G. A. J. Chem. Soc., Chem. Commun. 1973, 31-32.

(36) Yue, S.; Berry, G. C.; McCullough, R. D. Macromolecules 1996, 29, 933-939.

MA051122K 